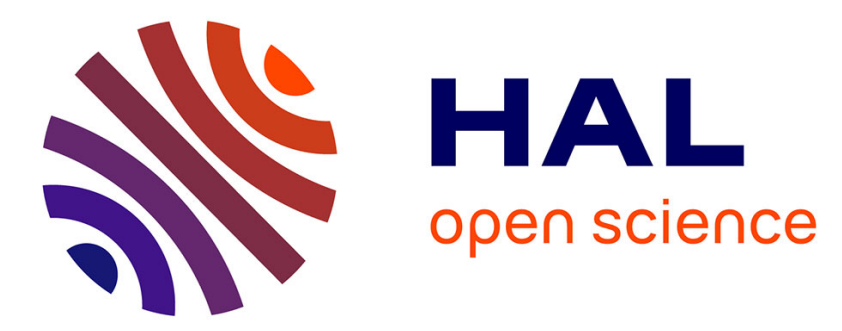

\title{
Equilibrium positions of composite dislocations in the f.c.c. structure
}

J. Bonneville, Joël Douin

\section{To cite this version:}

J. Bonneville, Joël Douin. Equilibrium positions of composite dislocations in the f.c.c. structure. Journal de Physique I, 1992, 2 (10), pp.1921-1928. 10.1051/jp1:1992255 jpa-00246672

\section{HAL Id: jpa-00246672 https://hal.science/jpa-00246672}

Submitted on 1 Jan 1992

HAL is a multi-disciplinary open access archive for the deposit and dissemination of scientific research documents, whether they are published or not. The documents may come from teaching and research institutions in France or abroad, or from public or private research centers.
L'archive ouverte pluridisciplinaire HAL, est destinée au dépôt et à la diffusion de documents scientifiques de niveau recherche, publiés ou non, émanant des établissements d'enseignement et de recherche français ou étrangers, des laboratoires publics ou privés. 


\title{
Equilibrium positions of composite dislocations in the f.c.c. structure
}

\author{
J. Bonneville and J. Douin (*) \\ Ecole Polytechnique Fédérale de Lausanne, I.G.A., CH 1015 Lausanne, Switzerland
}

(Received 7 February 1992, accepted in final form 23 June 1992)

\begin{abstract}
Résumé. - Dans le cadre de la théorie élastique isotrope, nous avons récemment montré qu'il est possible d'obtenir, à partir de l'énergie totale d'interaction entre les différentes partielles, une détermination analytique simple de la configuration d'équilibre de la dislocation de LomerCottrell. Nous avons ainsi établi que, contrairement à ce qui était généralement admis, celle-ci devait être asymétrique. En utilisant la même procédure, nous reconsidérons dans cet article les configurations d'équilibre de dissociation des différentes barrières de dislocations les plus probables dans la structure cubique à faces centrées $\left(B_{2}-B_{6}\right)$. Nous montrons que, comme pour la dislocation de Lomer-Cottrell, la configuration d'équilibre de la barrière $\mathrm{B}_{2}$ doit également être asymétrique. Les formes d'équilibres obtenues pour les autres barrières $\left(B_{3}-B_{6}\right)$ sont trouvées en bon accord avec l'ensemble des calculs antérieurs.
\end{abstract}

\begin{abstract}
In the frame-work of the isotropic elastic theory, we have recently shown that it is possible to derive, from the total interaction energy between the partials, a simple analytic solution for the equilibrium configuration of the Lomer-Cottrell dislocation. We have established, contrary to what is generally believed, that it should be asymmetric. By using the same procedure, we reconsider in this paper the equilibrium configurations of the extended dislocation barriers $\left(\mathbf{B}_{2}-\mathbf{B}_{6}\right)$ in the face centred cubic (f.c.c.) structure. As for the Lomer-Cottrell dislocation, the equilibrium configuration of the $B_{2}$ barrier must be also asymmetric. The equilibrium shapes obtained for the other barriers $\left(B_{3}-B_{6}\right)$ are found to be in good agreement with all previous predictions.
\end{abstract}

\section{Introduction.}

Composite dislocations are believed to play an important role in the plasticity of f.c.c. metals, and because they are sessile, they are often invoked in work-hardening theories [1-4]. Numerous observations of Lomer dislocations have been made with transmission electron microscopy (TEM) [5]. These dislocations are generally considered to be dissociated in a Lomer-Cottrell (or $B_{1}$, where $B_{1}-B_{6}$ refer to barriers as defined by Hirth and Lothe [6]) configuration because of their rectilinear character. Direct evidence of this dissociation was

(*) Now at: LEM, CNRS/ONERA, 29, av. de la Division Leclerc, 92322 Châtillon Cedex, France. 
supplied for instance by Korner and Karnthaler [7]. Other types of dissociated locks, such as the $B_{3}$, the $B_{4}[8]$, and the $B_{2}$ [9] have also been reported. Recent high resolution electron microscopy (HREM) observations have shown the $B_{2}$ core structure to be very compact in $\mathrm{Ni}_{3} \mathrm{Al}[10]$ and asymmetric in silicon [11].

It was generally well accepted that among the six most probable barriers, the $B_{1}-B_{4}$ were symmetric while the $B_{5}$ and $B_{6}$ were asymmetric [6]. However, in the framework of the anisotropic elasticity theory, Korner et al. [12] have shown that, contrary to previous investigations [13-16], the split configuration of the $B_{1}$ and $B_{2}$ locks in the f.c.c. structure should also be asymmetric.

In a more recent study [17], using isotropic elasticity, we have demonstrated that the $B_{1}$ dislocation is also asymmetric with a ratio $\delta$ between the two fault widths which is material independent. These results were found to be in good agreement with the anisotropic calculations [12]. In addition, the mean dissociation widths calculated by both theories were observed to be proportional to each other, for a number of pure metals and alloys, with stacking fault energies ranging between 5.6 and $125 \mathrm{~mJ} / \mathrm{m}^{2}$.

The purpose of this paper is to apply the same simple method of calculation [17] proposed for the $B_{1}$, to predict the isotropic splitting configuration of the five other barriers $\left(B_{2}-B_{6}\right)$ in the f.c.c. structure, and to compare the results of this new theoretical approach with the other calculations available in the literature.

\section{Method of calculation.}

Although extended locks involving extrinsic stacking faults have been recently observed in the $\mathrm{Ll}_{2}$ structure $[10,18]$, we consider throughout this study, as have all previous papers, only dislocation barriers extended in such a way that intrinsic stacking faults are formed. It must also be emphasized that, since, in this study, we are only interested in the equilibrium configuration of the dissociated barriers, only the interaction energy terms are relevant. Then the self energy terms need not to be considered.

All the investigated barriers have a three-fold non-planar configuration with two Shockley partials (1) and (2), which bound intrinsic stacking faults on two distinct $\{111\}$ planes with a common stair-rod (3) lying at the intersection line of the $\{111\}$ planes (Fig. 1). Using the abbreviated vector notation of Thompson [19] to denote the different Burgers vectors, the six most probable extended barriers can be written as :
1. $\mathrm{B} \delta(\mathrm{d})+\delta \mathrm{A}(\mathrm{d})+\mathrm{D} \alpha(\mathrm{a})+\alpha \mathrm{B}(\mathrm{a}) \rightarrow \delta \mathrm{A}+\mathrm{D} \alpha+\alpha \delta$
(acute)
2. $\mathrm{B} \delta(\mathrm{d})+\delta \mathrm{A}(\mathrm{d})+\mathrm{D} \alpha(\mathrm{a})+\alpha \mathrm{C}(\mathrm{a}) \rightarrow \mathrm{B} \delta+\alpha \mathrm{C}+\delta \mathrm{D} / \mathrm{A} \alpha$
3. $\mathrm{B} \delta(\mathrm{d})+\delta \mathrm{A}(\mathrm{d})+\mathrm{C} \alpha(\mathrm{a})+\alpha \mathrm{D}(\mathrm{a}) \rightarrow \delta \mathrm{A}+\alpha \mathrm{D}+\mathrm{BC} / \alpha \delta$
(obtuse)
4. $\mathrm{B} \delta(\mathrm{d})+\delta \mathrm{A}(\mathrm{d})+\mathrm{D} \alpha(\mathrm{a})+\alpha \mathrm{B}(\mathrm{a}) \rightarrow \alpha \mathrm{B}+\mathrm{B} \delta+\delta \mathrm{D} / \mathrm{A} \alpha$
(acute)
5. $\mathrm{B} \delta(\mathrm{d})+\delta \mathrm{A}(\mathrm{d})+\mathrm{D} \alpha(\mathrm{a})+\alpha \mathrm{C}(\mathrm{a}) \rightarrow \delta \mathrm{A}+\alpha \mathrm{C}+\mathrm{BD} / \delta \alpha$
(obtuse)
6. $\mathrm{B} \delta(\mathrm{d})+\delta \mathrm{A}(\mathrm{d})+\mathrm{B} \gamma(\mathrm{c})+\gamma \mathrm{D}(\mathrm{c}) \rightarrow \mathrm{B} \delta+\mathrm{B} \gamma+\delta \gamma / \mathrm{AD}$
(obtuse).

When the two Shockley partials are also considered as straight and parallel to the stair-rod, the total interaction energy can be expressed as :

$$
E_{\mathrm{nt}}=\sum_{i<j} E_{I j}+\gamma\left(d_{13}+d_{23}\right)
$$

where $\gamma$ is the intrinsic stacking fault energy and $E_{i j}$ the interaction energy per unit length between two parallel straight dislocations $i$ and $j$. In isotropic elasticity theory, $E_{l j}$ is given by [20] :

$$
E_{l j}=-\frac{\mu a^{2}}{2 \pi}\left(\alpha_{i j}+\frac{\beta_{i \jmath}}{(1-\nu)}\right) \log \frac{d_{i j}}{R}-\frac{\mu a^{2}}{2 \pi(1-\nu)} \psi_{i j}
$$



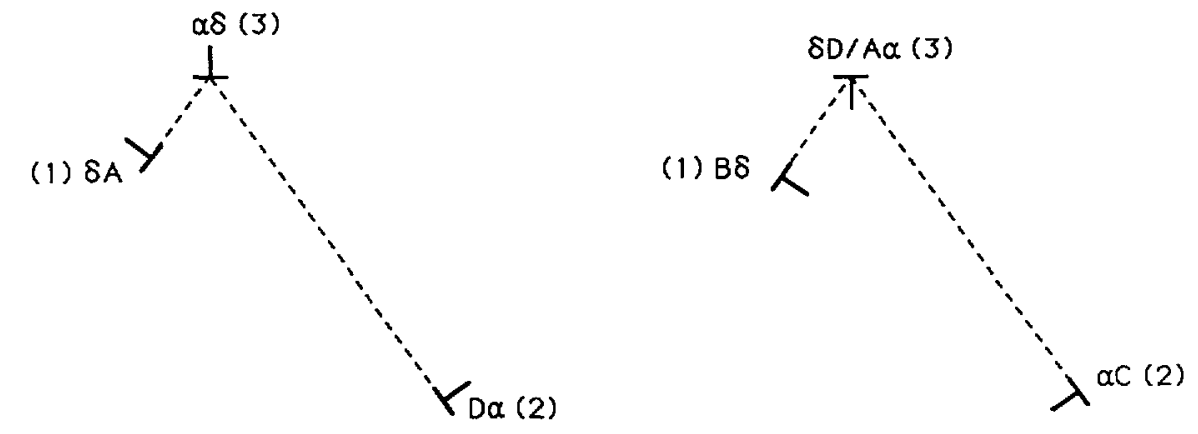

B1
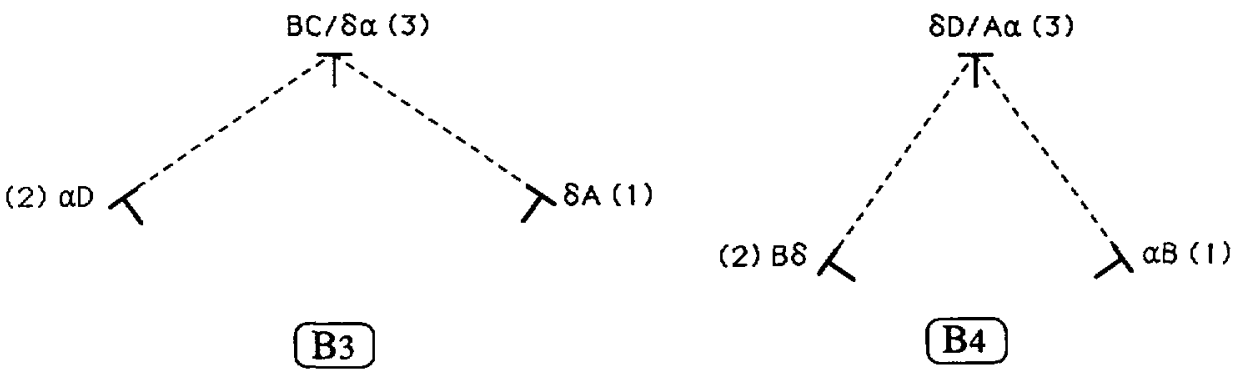

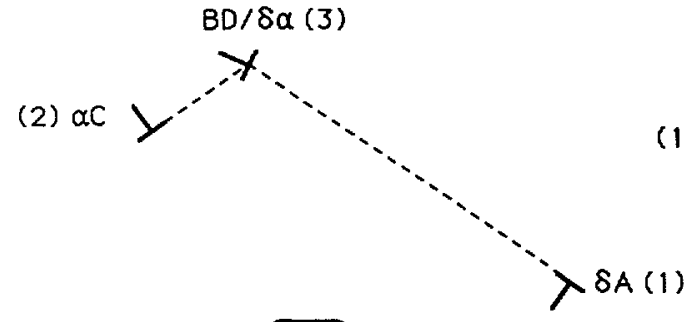

B5

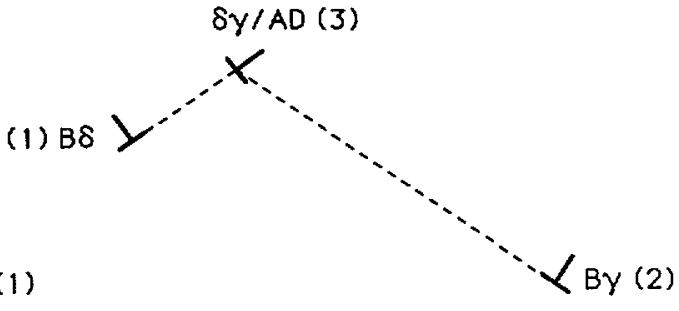

B6

Fig. 1. - Schematic diagram of the extended barriers $\left(B_{1}-B_{6}\right)$.

with,

$$
\begin{aligned}
& \alpha_{i j}=\left(\mathbf{b}_{\imath} \cdot \mathbf{t}\right)\left(\mathbf{b}_{j} \cdot \mathbf{t}\right) / a^{2} \quad \beta_{i j}=\left(\mathbf{b}_{i} \wedge \mathbf{t}\right) \cdot\left(\mathbf{b}_{j} \wedge \mathbf{t}\right) / a^{2} \\
& \psi_{i j}=\lambda_{i j} f(i, j)=\left[\left(\mathbf{b}_{\imath} \wedge \mathbf{t}\right) \cdot \mathbf{d}_{\imath j}\right]\left[\left(\mathbf{b}_{j} \wedge \mathbf{t}\right) \cdot \mathbf{d}_{\imath j}\right] / a^{2} d_{i j}^{2}
\end{aligned}
$$

and where $\mu$ and $\nu$ are the shear modulus and Poisson's ratio, respectively (for our isotropic approximation we take $\mu=C_{44}$ and $\left.\nu=1 / 3\right), \mathbf{b}_{i}$ is the Burgers vector of the dislocation $i, \mathbf{t}$ a unit vector along the dislocation line (here the intersection line of the two $\{111\}$ planes), $d_{i j}$ the separation distance between the dislocations $i$ and $j, R$ the outer cut-off radius, and a the 
lattice parameter. Table I lists the various $i j$ coefficients for the six different barriers, $B_{1}-B_{6}$, where the indices (1,2 and 3 ) have been assigned to the Burgers vectors of the composite dislocations in the same order as they appear in Thompson's notation above.

Table I. - The $i j$ coefficients of the dislocation barriers, $\mathbf{B}_{1}-\mathrm{B}_{6}\left(\lambda_{13}=\lambda_{23}=0, \forall B_{1}\right)$.

\begin{tabular}{|c|c|c|c|c|c|c|}
\cline { 2 - 7 } \multicolumn{1}{c|}{} & $\mathrm{B}_{1}$ & $\mathrm{~B}_{2}$ & $\mathrm{~B}_{3}$ & $\mathrm{~B}_{4}$ & $\mathrm{~B}_{5}$ & $\mathrm{~B}_{6}$ \\
\hline$\alpha_{12}$ & 0 & $1 / 8$ & 0 & $-1 / 8$ & 0 & $1 / 8$ \\
\hline$\alpha_{13}$ & 0 & 0 & 0 & 0 & 0 & $1 / 8$ \\
\hline$\alpha_{23}$ & 0 & 0 & 0 & 0 & $1 / 8$ & $1 / 8$ \\
\hline$\beta_{12}$ & $-1 / 18$ & $-1 / 72$ & $1 / 18$ & $-1 / 72$ & $-1 / 36$ & $1 / 72$ \\
\hline$\beta_{13}$ & $1 / 18$ & $1 / 18$ & $1 / 9$ & $1 / 18$ & $1 / 36$ & $-1 / 72$ \\
\hline$\beta_{23}$ & $1 / 18$ & $1 / 18$ & $1 / 9$ & $1 / 18$ & $5 / 72$ & $5 / 72$ \\
\hline$\lambda_{12}$ & $-4 / 27$ & $-1 / 27$ & $-4 / 27$ & $-1 / 27$ & $2 / 27$ & $-1 / 27$ \\
\hline
\end{tabular}

In expression (1), $E_{\text {int }}$ is actually only a function of the two splitting distances $d_{13}$ and $d_{23}$ because $d_{12}$ can be geometrically expressed in terms of $d_{13}$ and $d_{23}$ by :

$$
d_{12}=\sqrt{d_{13}^{2}+d_{23}^{2}-2 d_{13} d_{23} \cos \theta}
$$

with $\theta=70^{\circ} 53^{\prime}$ or $109^{\circ} 47^{\prime}$ for an acute or an obtuse extended barrier, respectively. As a consequence of this relationship, the equilibrium positions are defined by a system of two simultaneous polynomial equations :

$$
\left.\begin{array}{l}
\left(\frac{\partial E_{\mathrm{1nt}}}{\partial d_{23}}\right)_{d_{13}}=0 \\
\left(\frac{\partial E_{\mathrm{nt}}}{\partial d_{13}}\right)_{d_{23}}=0
\end{array}\right\}
$$

which, in general, in this form does not have explicit solutions. However, as was already reported in [17], it is possible to find a more appropriate expression of $E_{1 \mathrm{mt}}\left(d_{13}, d_{23}\right)$ by assigning to $d_{13}$ and $d_{23}$ two new variables $d$ and $\xi$, defined as (1):

$$
d_{13}=d(1-\xi)=d f_{13}(\xi) \text { and } d_{23}=d(1+\xi)=d f_{23}(\xi)
$$

(1) The solution $d_{13}=d(1+\xi)$ and $d_{23}=d(1-\xi)$ is also valid. Thus, $\xi=0$ signifies a symmetrically dissociated barrier $\left(d=d_{12}=d_{23}\right.$ ), while any value of $\xi \neq 0$ corresponds to an asymmetric barrier $\left(d \neq d_{12} \neq d_{23}\right)$. It should be noted that for all barriers both solutions, i.e. symmetric or asymmetric, the total stacking fault width is the same $\left(d_{12}+d_{23}\right)_{\text {asymm }}=\left(d_{12}+d_{23}\right)_{\text {symm }}$. In the following, $\xi$ will be referred to as the « asymmetry » parameter. 
which lead to :

$$
d_{12}=d f_{12}(\xi)
$$

where $f_{12}(\xi)$ is dependent on the geometry of the barrier and equal to $2 \sqrt{\left(1+2 \xi^{2}\right) / 3}$ or $2 \sqrt{\left(2+\xi^{2}\right) / 3}$ for an acute or an obtuse extended barrier, respectively. Then, it is easy to show from (1), (2), (5) and (6) that $E_{\mathrm{int}}(d, \xi)$ can be decomposed into two separate functions of $d$ and $\xi$, namely :

$$
E_{\mathrm{int}}=f_{1}(d)+f_{2}(\xi)+\mathrm{C}^{\mathrm{st}}
$$

with :

$$
\begin{aligned}
& f_{1}(d)=-\frac{\mu a^{2}}{2 \pi}\left[\sum_{i<j}\left(\alpha_{i j}+\frac{\beta_{i j}}{1-\nu}\right)\right] \log \frac{d}{R}+2 \gamma d \\
& f_{2}(\xi)=-\frac{\mu a^{2}}{2 \pi} \sum_{i<j}\left[\left(\alpha_{i j}+\frac{\beta_{i_{j}}}{1-\nu}\right) \log \left(f_{i j}(\xi)\right)+\frac{\psi_{i j}(\xi)}{1-\nu}\right]
\end{aligned}
$$

and where $C^{\text {st }}$ is a constant term. With this new expression of $E_{1 \mathrm{nt}}$, it is clear that the mean splitting distance, $d=\left(d_{13}+d_{23}\right) / 2$, of the extended barrier can be deduced from the $f_{1}(d)$ function independently of the « asymmetry » parameter, $\xi=\left(d_{23}-d_{13}\right) /\left(d_{13}+d_{23}\right)$, which can be obtained by studying the $f_{2}(\xi)$ function, only. In expression (9), the $\psi_{i}$ term which is $\xi$ dependent, is :

$$
\psi_{12}(\xi)=\lambda_{12} \frac{f_{13}(\xi) f_{23}(\xi)}{f_{12}^{2}(\xi)}
$$

Then, the equilibrium positions can be determined by solving the equivalent set of equations :

$$
\left.\begin{array}{l}
\left(\frac{\partial E_{\mathrm{int}}}{\partial d}\right)_{\xi}=0 \Rightarrow\left(\frac{\partial f_{1}(d)}{\partial d}\right)=0 \\
\left(\frac{\partial E_{\mathrm{nt}}}{\partial \xi}\right)_{d}=0 \Rightarrow\left(\frac{\partial f_{2}(\xi)}{\partial \xi}\right)=0
\end{array}\right\}
$$

which gives the general expressions of the equilibrium parameters, $d_{\mathrm{e}}$ and $\xi_{\mathrm{e}}$.

$$
d_{\mathrm{e}}=\frac{\mu a^{2}}{4 \pi \gamma}\left[\sum_{1<j}\left(\alpha_{\imath \jmath}+\frac{\beta_{1 \jmath}}{1-\nu}\right)\right]=\frac{\mu a^{2}}{4 \pi \gamma} \eta
$$

and

$$
-\sum_{i<J}\left[\left(\alpha_{i j}+\frac{\beta_{i j}}{1-\nu}\right) \frac{f_{i j}^{\prime}\left(\xi_{\mathrm{e}}\right)}{f_{\imath J}\left(\xi_{\mathrm{e}}\right)}+\frac{\psi_{i j}^{\prime}\left(\xi_{\mathrm{e}}\right)}{1-\nu}\right]=0
$$

where the prime denotes the first derivative of the corresponding function with respect to $\xi$.

Equation (13) leads to a fifth-degree polynomial with solutions that can be accurately determined by using a root-finder computer program. Depending on the extended barrier, the number of real solution(s) is one or three ; if it is three, two of them are symmetrical. Two sets of complex conjugate roots exist when there is only one real solution and only one set accompanies the case where there are three real solutions. When more than one real solution 
exists, the stable equilibrium positions can easily be found by studying the sign of the function $f_{2}^{\prime}(\xi)$ or by plotting the $f_{2}(\xi)$ function.

It is also directly observable from (12) that in isotropic elasticity the " asymmetry " parameter $\xi_{\mathrm{e}}$, which determines the positions of the Shockley partials relative to the mean splitting width $\left(d_{\mathrm{e}}\right)$, is $\mu$ independent and is only material dependent through Poisson's ratio. Furthermore, it should be noted that, since $\alpha_{I J}=0 \forall(i, j)$ for the $\mathbf{B}_{1}$ and $\mathbf{B}_{3}$ configurations, the $\xi_{\mathrm{e}}$ values of these two barriers are $\nu$ independent, i.e. material-independent.

\section{Results and discussion.}

For the sake of simplicity and because we are mainly interested in comparing the pure isotropic case to earlier results, as was done in [17], the $\eta$ coefficients and the equilibrium $\xi_{\mathrm{e}}$ variables reported in table II have been calculated with respect to a Poisson ratio equal to $1 / 3$. For convenience, the ratios $\delta_{\mathrm{e}}=d_{23} / d_{13}$ are also given in this table using the positive values of $\xi_{\mathrm{e}}$ for the $\mathrm{B}_{1}$ and $\mathrm{B}_{2}$.

Table II. - The equilibrium position parameters $\eta, \xi_{\mathrm{e}}$ and $\delta_{\mathrm{e}}$ for the dislocation barriers, $\mathrm{B}_{1}-\mathrm{B}_{6}$.

\begin{tabular}{|c|c|c|c|c|c|c|}
\cline { 2 - 7 } \multicolumn{1}{c|}{} & $\mathrm{B}_{1}$ & $\mathrm{~B}_{2}$ & $\mathrm{~B}_{3}$ & $\mathrm{~B}_{4}$ & $\mathrm{~B}_{5}$ & $\mathrm{~B}_{6}$ \\
\hline$\eta(\times 48)$ & 4 & 13 & 20 & 1 & 11 & 23 \\
\hline$\xi_{\mathrm{e}}$ & \pm 0.585 & \pm 0.535 & 0 & 0 & 0.531 & 0.503 \\
\hline$\delta_{\mathrm{e}}$ & 3.82 & 3.3 & 1 & 1 & 3.26 & 3.02 \\
\hline
\end{tabular}

It must be emphasized that when $\xi_{\mathrm{e}}$ is $\nu$ dependent (i.e. the $\mathrm{B}_{2}, \mathrm{~B}_{5}$ and $\mathrm{B}_{6}$ ) its dependence is always very small with respect to this last elastic constant. For instance, taking $\nu=0.254(\mathrm{Th})$ and $\nu=0.412(\mathrm{Au})$ lead in the case of the $\mathrm{B}_{6}$, which is the most $\nu$ sensitive, to $\xi_{\mathrm{e}}=0.468$ and 0.534 , respectively. On the opposite, the $\eta$ coefficient can vary more significantly with $\nu$, especially for the $\mathrm{B}_{4}$ where using the above $\nu$ values will correspond to an increase of $\eta$ by a factor of almost 4 .

In contrast to previous investigations that were based on both isotropic [14-16] and anisotropic elasticity $[13,14]$, the results listed in table II indicate that the $B_{1}$ and the $B_{2}$ extended barriers will dissociate in an asymmetrical configuration. It is important to recognize that the symmetric solution, $\xi_{e}=0$, does exist for these two barriers but corresponds to an unstable position of a local maximum of the interaction energy $\left(E_{\mathrm{mt}}\right)$ as is shown in figure 2 . The plus-minus sign in table II indicates that both solutions are valid and lead to the same minimum of $E_{\mathrm{nt}}$. Thus, in this case the extended barrier can occur into two asymmetric forms with an inverse ratio between the two arms.

These results are in good agreement with the anisotropic calculation of Korner et al. [12]. However, it is necessary to distinguish the two barriers since the fair quantitative agreement obtained for the $\mathrm{B}_{1}$ [17] does not hold for the $\mathrm{B}_{2}$. Indeed, using the $\mu=C_{44}$ values of [12], we predict a mean dissociation width $d_{e}$ approximately twice that obtained by anisotropic computation (see Fig. 3), while $\xi_{\mathrm{e}}$ is also found to be rather different for the two theories. The $\xi_{\mathrm{e}}$ isotropic value of 0.535 , or equivalently $\delta_{\mathrm{e}}=3.3$, is compared with those obtained by Korner et al. [12] in table III. 


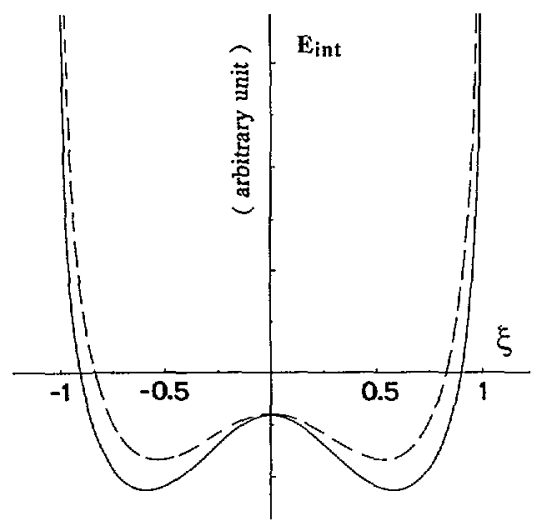

Fig. 2.

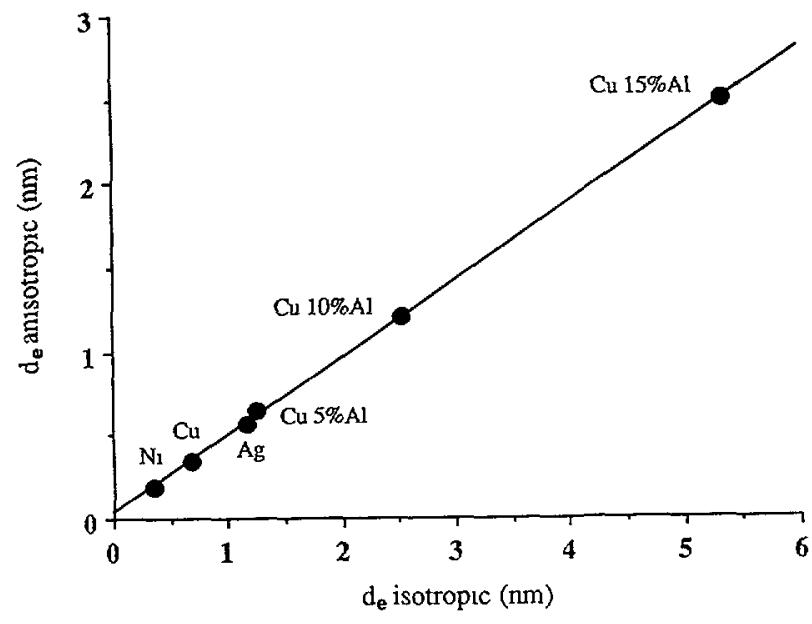

Fig. 3.

Fig. 2. - Shape of the interaction energy, $E_{\text {int }}$, as a function of the " asymmetry » parameter, $\xi$, for the $\mathrm{B}_{1}$ (solid line) and the $\mathrm{B}_{2}$ (dashed line). (Note : the curves have been shifted along the $E_{\mathrm{lnt}}$ axis and the values of $E_{\mathrm{nt}}$ at $\xi=0$ are not actually equivalent.)

Fig. 3. - Isotropic vs. anisotropic theoretical mean dissociation widths $\left(d_{c}\right)$ for the $\mathrm{B}_{2}$. Anisotropic mean values have been obtained from table 4 of [12].

Table III. - The anisotropic $\xi_{\mathrm{e}}$ asymmetry parameters and $\delta_{\mathrm{e}}$ ratios of the $\mathrm{B}_{2}$ lock according to Korner et al. [12].

\begin{tabular}{|c|c|c|c|c|c|c|}
\cline { 2 - 7 } \multicolumn{1}{c|}{} & $\mathrm{Cu}$ & $\mathrm{Cu} 5 \% \mathrm{Al}$ & $\mathrm{Cu} 10 \% \mathrm{Al}$ & $\mathrm{Cu} 15 \% \mathrm{Al}$ & $\mathrm{Ag}$ & $\mathrm{Ni}$ \\
\hline$\xi_{\mathrm{e}}$ & 0.291 & 0.270 & 0.245 & 0.225 & 0.293 & 0.369 \\
\hline$\delta_{\mathrm{e}}$ & 1.82 & 1.74 & 1.65 & 1.58 & 1.83 & 2.17 \\
\hline
\end{tabular}

For the other four barriers, $\mathrm{B}_{3}$ to $\mathrm{B}_{6}$, the $\xi_{\mathrm{e}}$ solution is unique (see Tab. II). The $\mathrm{B}_{3}$ and the $B_{4}$ are found to be symmetrically dissociated while the $B_{5}$ and the $B_{6}$ are asymmetric in agreement with all the preceding predictions [12-16]. Furthermore, as was proposed by [14], using the most accurate values for the shear modulus and Poisson's ratio given in [21] yields the same isotropic equilibrium parameters reported in table I of [14]. For these barriers, the comparison developed in [14] between the isotropic and anisotropic results should be valid.

\section{Conclusions.}

We have shown that by choosing a suitable set of variables it is possible to minimize the total interaction energy of three-fold composite dislocations. This approach, which is based on isotropic elasticity theory, allows for the straightforward derivation of an analytical solution for the equilibrium positions of the partials. By using this change of variables, we have been able to prove that for the $B_{1}$ and $B_{2}$ barriers the symmetrical configuration is metastable and only corresponds to a local maximum of the interaction energy.

It has been reported that the results based on the isotropic theory and those based on the anisotropic theory may differ by a negligible amount [22]. Our previous results [17] and the 
present study both suggest that among the six most probable locks of the fcc structure a fair quantitative agreement only exists for the $B_{1}$. The agreement is only qualitative for the other five barriers. Thus, only in the particular case of the $\mathbf{B}_{1}$ can the anisotropic dissociation widths be estimated by using the isotropic relations and the small correction term proposed in figure 2 of [17].

Finally, we would like to conclude with two general remarks :

i) The use of the proposed variable transformations is not restricted to the composite dislocations of this study, but can be applied to other three-fold dissociation configurations. This should lead to equivalent forms of expressions (12) and (13) for the equilibrium positions, in a way that is much easier than solving the equilibrium set of equations (4).

ii) Only local minima can be mathematically determined. More precisely, the point where the derivative of a function goes to zero does not necessarily correspond to a minimum in this function. Computations of partial equilibrium positions of composite dislocations that are simply based on the fact that the sum of all forces on each partial is zero can lead to erroneous results. As shown in this study, this does not always correspond to a stable minimum of the total interaction energy.

\section{Acknowledgments.}

The authors are especially grateful to Dr. K. J. Hemker, Professor J. L. Martin and Professor $\mathrm{G}$. Vanderschaeve for stimulating discussions and for valuable comments about the manuscript. Financial support for this study was provided by Fonds National Suisse.

\section{References}

[1] Friedel J., Philos. Mag. 46 (1955) 1169.

[2] SaAda G., Acta Metall. 8 (1960) 841.

[3] Seeger A., Encyclopedia of Physics, Vol. 7/2 (Berlin : Springer-Verlag, 1958).

[4] Hirsch P. B. and Mitchell T. E., Can. J. Phys. 45 (1967) 663.

[5] BASINSKi S. J. and BASINSKi Z. S., Dislocations in Solids 4 (1979) 261.

[6] HirTh J. P. and Lothe J., Theory of Dislocations (John Wiley \& Sons, New York, 1982) p. 800.

[7] Korner A. and Karnthaler H. P., Philos. Mag. 44 (1981) 275.

[8] Karnthaler H. P. and Wintner E., Acta Metall. 23 (1975) 1501.

[9] Skalicky P., Phys. Stat. Sol. (a) 20 (1973) 11.

[10] Baluc N., PhD Thesis n 886, Ecole Polytechnique Fédérale de Lausanne, Switzerland (1990).

[11] Elkajbaji M., Kirchner H. O. and Thibault-Dessaux J., Philos. Mag. A 57 (1988) 631.

[12] Korner A., SChmid H. and Prinz F., Phys. Stat. Sol. (a) 51 (1979) 613.

[13] Teutonico L. J., Philos. Mag. 10 (1964) 401.

[14] Jøssang T., Hirth J. P. and Hartley C. S., J. Appl. Phys. 36 (1965) 2400.

[15] HiRTh J. P., J. Appl. Phys. 32 (1961) 700.

[16] HiRTh J. P., J. Appl. Phys. 33 (1962) 2286.

[17] Bonneville J. and Douin J., Philos. Mag. Lett. 62 (1990) 247.

[18] Sun Y., PhD Thesis, University of Oxford (1990).

[19] Thompson N., Proc. Phys. Soc. London 66B (1953) 481.

[20] HiRTh J. P. and Lothe J., Theory of Dislocations (John Wiley \& Sons, New York, 1982) p. 117.

[21] VoIGT W., Lehrbuch der Kristalphysik, Leipzig (1928) 716.

[22] SPENCE G. B., J. Appl. Phys. 33 (1962) 729. 